\title{
Chemical abundance patterns of extremely metal-poor stars
}

\author{
Wako Aoki* \\ National Astronomical Observatory of Japan \\ E-mail: aoki.wako@nao.ac.jp
}

\section{Timothy C. Beers}

Department of Physics and Astronomy and JINA: Joint Institute for Nuclear Astrophysics, Michigan State University

E-mail: beers@pa.msu.edu

\section{Norbert Christlieb}

Hamburger Sternwarte, University of Hamburg

E-mail: nchristliebehs . uni-hamburg. de

\section{Anna Frebel}

Research School of Astronomy and Astrophysics, Australian National University

E-mail: anna@mso.anu.edu.au

\section{John E. Norris}

Research School of Astronomy and Astrophysics, Australian National University

E-mail: jen@mso.anu.edu.au

\section{Satoshi Honda}

National Astronomical Observatory of Japan

E-mail: honda@optik.mtk.nao.ac.jp

\section{Masahide Takada-Hidai}

Liberal Arts Education Center, Tokai University

E-mail: hidai@apus.rh.u-tokai.ac.jp

\section{Martin Asplund}

Research School of Astronomy and Astrophysics, Australian National University

E-mail: martin@mso.anu.edu.au

\section{Hiroyasu Ando}

National Astronomical Observatory of Japan

E-mail: ando@optik.mtk.nao.ac.jp

\section{Sean G. Ryan}

Department of Physics and Astronomy, Open University

E-mail: s.g.ryan@herts.ac.uk

\section{Stelios Tsangarides}




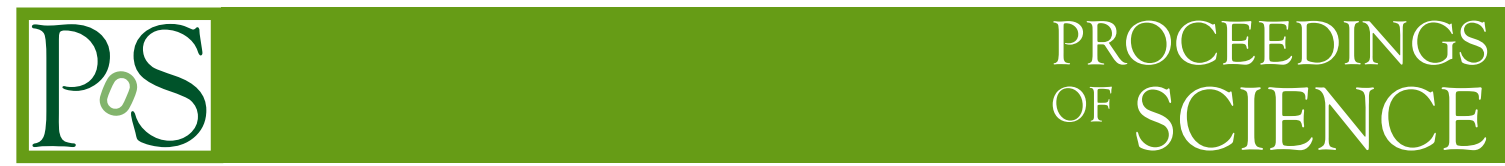

Department of Physics and Astronomy, Open University

E-mail: stsangarides@gmail.com

We have obtained high resolution $(R=60,000)$, high quality $(S / N>100)$ spectroscopy using Subaru/HDS for $\sim 20$ candidate extremely metal-poor stars $([\mathrm{Fe} / \mathrm{H}]<-3)$ to determine their chemical abundance patterns. In the observing program we found $\mathrm{HE} 1327-2326$, which has $[\mathrm{Fe} / \mathrm{H}]=-5.4$, the lowest Fe abundance known in normal stars. This star shows extremely large excesses of $\mathrm{C}$ and $\mathrm{N}$, and also excesses of other light elements (e.g. O, Na). The low iron abundance and the peculiar abundance pattern provide a signature of the nucleosynthesis by the first generation stars in the Universe. We also present the preliminary results for other stars: (1) The iron abundances of our sample, except for $\mathrm{HE} 1327-2326$, are higher than $[\mathrm{Fe} / \mathrm{H}]=-4$. There is a gap of iron abundance between $[\mathrm{Fe} / \mathrm{H}]=-4$ and -5 . This would indicate that the nucleosynthesis, or formation processes that are responsible for the stars with $[\mathrm{Fe} / \mathrm{H}]<-5$ and others are quite different. (2) Six stars of our sample, including HE1327-2326, show clear excesses of carbon. Only two of them show excesses of the heavy neutron-capture element $\mathrm{Ba}$, suggesting a large contribution of AGB nucleosynthesis. The origin of carbon-excesses in other stars are still unclear, but the existence of these stars is a remarkable feature only found at the extremely low metallicity.

International Symposium on Nuclear Astrophysics - Nuclei in the Cosmos - IX

June 25-30 2006

CERN, Geneva, Switzerland

* Speaker. 


\section{Introduction}

Measurements of chemical compositions for very metal-poor stars have been providing useful constraints on individual nucleosynthesis processes, because such stars are believed to have formed in the very early Galaxy, and the yields from individual objects like core-collapse supernovae have not yet been well mixed in interstellar matter. Abundance studies based on high resolution spectroscopy for very metal-poor stars in the past decade have revealed that the chemical abundance patterns of extremely metal-poor (EMP) stars, which have $[\mathrm{Fe} / \mathrm{H}]<-3^{1}[1]$, are sometimes quite different from those of stars with higher metallicity. One important result is that the fraction of carbon-enhanced objects is quite high in this metallicity range [2]. An extreme example is HE0107-5240, which is the most iron-deficient star known until 2004. This object shows significantly high overabundances of $\mathrm{C}, \mathrm{N}$, and $\mathrm{O}[3,4,5]$. A few objects also show large excesses of alpha elements $[6,7,8]$. Such abundance trends indicate objects in the lowest metallicity range include rich information for unknown nucleosynthesis processes in the very early Galaxy.

High resolution spectrographs mounted on 8-10m class telescopes (Keck, VLT, and Subaru) have been applied to measurements of chemical compositions of very metal-poor stars in the past several years, enlarging the sample of metal-poor stars for which detailed abundance patterns are measured $[9,10,11]$. However, measurements for the lowest metallicity range $([\mathrm{Fe} / \mathrm{H}]<-3)$ are still quite limited. This is because objects with such low metallicity are quite rare in the solar neighborhood, and the absorption lines in their spectra that are used for abundance measurements are very weak, requiring very high quality spectra for abundance measurements. In order to investigate the chemical abundance patterns of stars with $[\mathrm{Fe} / \mathrm{H}]<-3.0$, we have conducted a program to observe candidate EMP stars in 2003-2005. In the observing program, we successfully discovered the most iron-deficient star HE 1327-2326 [12, 13]. In this paper, we report the chemical composition of this object, and the preliminary results for other EMP stars.

\section{Observations}

Candidate metal-poor stars in our program were initially selected by the HK-survey [14] and the Hamburg/ESO survey [15]. The targets for high resolution spectroscopy were selected based on medium-resolution spectra for the above sample. Optical photometry was obtained for most of our targets to constrain the stellar parameters (e.g. effective temperatures) of the sample. Several objects were also selected from the HERES program [16].

High-resolution spectroscopy for our targets was obtained with the High Dispersion Spectrograph [17] of the Subaru Telescope. We have carried out four observing runs from December 2003 to June 2005, and have obtained spectra of $\sim 30$ stars with a resolving power of $R=60,000$ covering 4000-6800 $\AA$, among which spectra of $\sim 20$ stars have sufficient signal-to-noise ratios (100/1 at $4500 \AA$ ). The observations of HE 1327-2326 were made in May and June, 2004. For other objects, we here discuss preliminary results on the chemical abundances of our targets observed until February 2005.

\footnotetext{
${ }^{1}[\mathrm{~A} / \mathrm{B}]=\log \left(N_{\mathrm{A}} / N_{\mathrm{B}}\right)-\log \left(N_{\mathrm{A}} / N_{\mathrm{B}}\right) \odot$ for elements $\mathrm{A}$ and $\mathrm{B}$.
} 


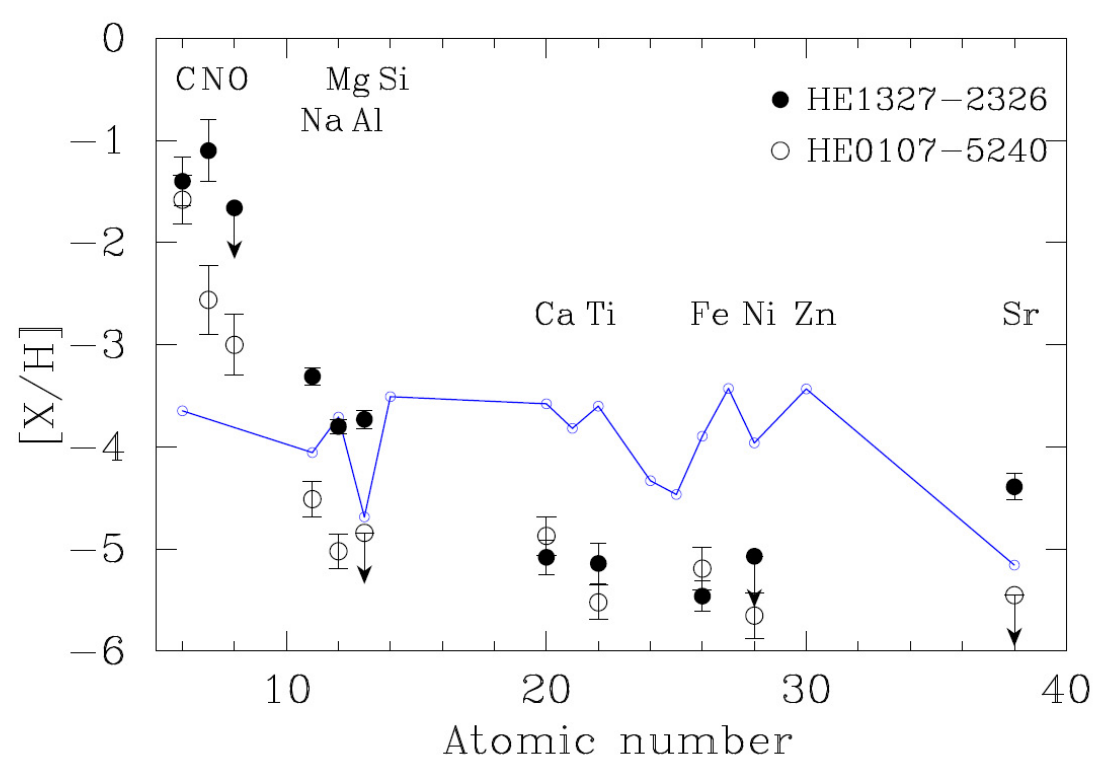

Figure 1: Chemical abundance patterns of HE 1327-2326 (filled circles) and HE 0107-5240 (open circles). The line indicates the abundance pattern of the average of four EMP stars (see text).

\section{Chemical compositions of HE 1327-2326, the most iron-deficient star known}

Figure 1 shows the chemical abundances of HE 1327-2326 and HE 0107-5240, compared with the average of the abundance patterns of the other EMP stars (CD - 38 245, CS 22885-096, BS 16467-062, and CS 22172-002: [18, 9]). These two stars have outstandingly lower abundances of iron-peak elements than those of other EMP stars. The iron abundance of HE 1327-2326 is slightly lower than HE 0107-5240, and is about two orders of magnitude lower than the iron abundance of the previously known most iron-deficient main-sequence star CS 22876-032. By contrast, the two objects have large overabundances of carbon $([\mathrm{C} / \mathrm{Fe}]=+4)$.

The low Fe abundance and the excess of carbon in HE 1327-2326 and HE 0107-5240 suggest that the abundance patterns of these two stars have a similar origin. Despite their overall similarity, significant differences in the abundance patterns are found for several of the light elements in these two stars. The nitrogen abundance of HE $1327-2326([\mathrm{~N} / \mathrm{Fe}] \sim+4)$ is remarkably higher than that of HE 0107-5240. The abundances of $\mathrm{Na}, \mathrm{Mg}$, and $\mathrm{Al}$ of HE 1327-2326 are also about 1 dex higher than those of HE 0107-5240 (see Figure 1). This result suggests that a large variation is allowed in the yields of the elements from $\mathrm{N}$ to $\mathrm{Al}$ in the nucleosynthesis processes that preceded the formation of these two stars.

In order to interpret the peculiar abundance patterns of HE 1327-2326 and HE 0107-5240, several models have been proposed. One idea is to assume that this object belongs to a binary system, and that mass transfer from the primary star in its Asymptotic Giant Branch (AGB) phase has produced the elements now observed on the surviving companion [19]. This model explains the large excesses of carbon and other light elements, while accretion of iron-peak elements from interstellar medium should be assumed. It should be noted that there is still no evidence for binarity 
for HE 1327-2326 nor HE 0107-5240.

Other models assume that the abundance patterns of these two stars were produced by massive progenitor stars. Umeda \& Nomoto [20] proposed a so-called "faint supernova" model, which well reproduces the abundance pattern of HE 0107-5240. The model was updated by Iwamoto et al. [21] to reproduce the abundance pattern of HE 1327-2326. The significant difference of the $\alpha / \mathrm{Fe}$ ratios between the two stars is explained by the small difference of the mixing region and fallback assumed in their models. Another model is discussed by Meynet et al. [22], who predict that large mass loss, including significant amounts of light elements, may have occurred in massive rotating stars with very low metallicity.

One unexpected result is that the resonance line of neutral $\mathrm{Li}$ at $6708 \AA$, which is found in most metal-poor stars with sufficiently high effective temperatures $\left(T_{\text {eff }}>6000 \mathrm{~K}\right)$, is not detected in HE 1327-2326. The Li abundances of metal-poor dwarf/subgiant stars produce the socalled Spite plateau at $\log \varepsilon(\mathrm{Li}) \sim 2.2$. However, the observed upper limit on the $\mathrm{Li}$ abundance of HE $1327-2326$ is $\log \varepsilon(\mathrm{Li}) \sim 1.5$. This indicates that some unidentified mechanism may have depleted the $\mathrm{Li}$ in the atmosphere of this object, one that does not operate for most metal-deficient subgiants and dwarfs. Quite recently, Piau et al. [23] proposed a possibility that a significant amount of primordial material was processed by the first generations of massive stars, and HE 1327-2326 formed from the cloud significantly contaminated by the ejecta from such objects. Further measurements of $\mathrm{Li}$ abundances for extremely metal-poor dwarf/subgiant stars are clearly desired to examine such scenarios.

Another unexpected result is the detection of $\mathrm{Sr}$, a species located near the first abundance peak of neutron-capture elements. The origin of this element is discussed by Aoki et al. [24] separately.

\section{Preliminary results for other EMP stars}

The abundance analyses for our sample identified 15 stars to have $[\mathrm{Fe} / \mathrm{H}]<-3$ to date. Most of them have $-3.5<[\mathrm{Fe} / \mathrm{H}]<-3.0$, suggesting the rapid decline of the number of objects with decreasing metallicity. In particular, there is no object in $-5<[\mathrm{Fe} / \mathrm{H}]<-4$, while two stars (HE 1327-2326 and HE 0107-5240) are known to have $[\mathrm{Fe} / \mathrm{H}]<-5$. This gap in the ironabundances could have important implications on the formation mechanisms of the most metaldeficient stars. The large overabundances of carbon in the two stars with $[\mathrm{Fe} / \mathrm{H}]<-5$, which cannot be attributed to any biases in the sample selection, provide another key to modeling the nucleosynthesis processes responsible for these two stars $[12,13]$.

One remarkable observational fact found for EMP stars is that the fraction of carbon-enhanced stars is quite high. Aoki et al. [25] showed that some fraction of carbon-enhanced EMP stars show no excess of neutron-capture elements, while all objects having higher metallicity $([\mathrm{Fe} / \mathrm{H}]>-2.6)$ exhibit large excesses of s-process elements. Our sample includes six carbon enhanced $([\mathrm{C} / \mathrm{Fe}] \gtrsim$ $+1)$ stars, including HE 1327-2326. Two stars at $[\mathrm{Fe} / \mathrm{H}] \sim-3$ with a very large enhancement of carbon $([\mathrm{C} / \mathrm{Fe}]>+2)$ also show an excess of the s-process element $\mathrm{Ba}$. The $\mathrm{C}$ overabundances are interpreted as the result of AGB nucleosynthesis and mass transfer across binary systems. The other four stars show no clear excesses of neutron-capture elements. The present study confirms the suggestion from previous work that a large fraction of EMP stars have overabundances of carbon, 
and contribution of the carbon production in some objects other than AGB stars is significant in the lowest metallicity range.

The observing program of EMP stars with Subaru/HDS has already been completed. Another observing program for candidate EMP stars accessible from the southern hemisphere using VLT/UVES is ongoing. The full abundance analyses for the data set will provide useful constraints on the models of nucleosynthesis in the first generations of stars.

\section{References}

[1] Beers, T. C., Christlieb, N. 2005, The Discovery and Analysis of Very Metal-Poor Stars in the Galaxy, Annual Review of Astronomy and Astrophysics $\mathbf{4 3} 531$

[2] Beers, T. C., The frequency of carbon-enhanced stars in HERES and SDSS, this volume

[3] Christlieb, N., Bessell, M. S., Beers, T. C., Gustafsson, B., Korn, A., Barklem, P. S., Karlsson, T., Mizuno-Wiedner, M., Rossi, S. 2002, A stellar relic from the early Milky Way, Nature 419904

[4] Bessell et al. 2004 Bessell, M., Christlieb, N., \& Gustafsson, B. 2004, Astrophysical Journal 612 L61

[5] Christlieb, N., Gustafsson, B., Korn, A. J., Barklem, P. S., Beers, T. C., Bessell, M. S., Karlsson, T., \& Mizuno-Wiedner, M. 2004, Astrophysical Journal 603708

[6] Norris, J. E., Ryan, S. G., \& Beers, T. C. 2001, Astrophysical Journal 5611034

[7] Depagne, E., Hill, V., Spite, M., Spite, F., Plez, B., Beers, T. C., Barbuy, B., Cayrel, R., Andersen, J., Bonifacio, P., François, P., Nordström, B., \& Primas, F. 2002, Astronomy \& Astrophysics 390187

[8] Aoki, W., Norris, J. E., Ryan, S. G., Beers, T. C., \& Ando, H. 2002, Astrophysical Journal 576 L141

[9] Cayrel, R. et al. 2004, First stars V - Abundance patterns from $C$ to Zn and supernova yields in the early Galaxy. Astronomy and Astrophysics 416, 1117

[10] Honda, S., Aoki, W., Kajino, T., Ando, H., Beers, T. C., Izumiura, H., Sadakane, K., Takada-Hidai, M. 2004, Spectroscopic Studies of Extremely Metal-Poor Stars with the Subaru High Dispersion Spectrograph. II. The r-Process Elements, Including Thorium, Astrophysical Journal 607474

[11] Cohen, J. G., Christlieb, N., McWilliam, A., Shectman, S., Thompson, I., Wasserburg, G. J., Ivans, I., Dehn, M., Karlsson, T., \& Melendez, J. 2004, Astrophysical Journal 6121107

[12] Frebel, A. et al. 2005, Nucleosynthetic signatures of the first stars, Nature $\mathbf{4 3 4} 871$

[13] Aoki, W. et al. 2006, HE 1327-2326, an Unevolved Star with [Fe/H] $<-5.0$. I. A Comprehensive Abundance Analysis, Astrophysical Journal 639897

[14] Beers, T. C., Preston, G. W., \& Shectman, S. A. 1992, Astronomical Journal 1031987

[15] Christlieb, N. 2003, Finding the Most Metal-poor Stars of the Galactic Halo with the Hamburg/ESO Objective-prism Survey, Reviews of Modern Astronomy 16191

[16] Barklem, P. S., Christlieb, N., Beers, T. C., Hill, V., Bessell, M. S., Holmberg, J., Marsteller, B., Rossi, S., Zickgraf, F.-J., Reimers, D. 2005. The Hamburg/ESO R-process enhanced star survey (HERES). II. Spectroscopic analysis of the survey sample, Astronomy and Astrophysics $\mathbf{4 3 9} 129$

[17] Noguchi, K., Aoki, W., Kawanomoto, S., et al. 2002, High Dispersion Spectrograph for the Subaru Telescope, Publication of Astronomical Society of Japan $\mathbf{5 4} 855$ 
[18] François, P., Depagne, E., Hill, V., Spite, M., Spite, F., Plez, B., Beers, T., Barbuy, B., Cayrel, R., Andersen, J., Bonifacio, P., Molaro, P., Nordström, B., \& Primas, F. 2003, Astronomy and Astrophysics $\mathbf{4 0 3} 1105$

[19] Suda, T., Aikawa, M., Machida, M. N., Fujimoto, M. Y., \& Iben, I. J. 2004, Astrophysical Journal 611 476

[20] Umeda, H., Nomoto, K. 2003, First-generation black-hole-forming supernovae and the metal abundance pattern of a very iron-poor star, Nature $\mathbf{4 2 2} 871$

[21] Iwamoto, N., Umeda, H., Tominaga, N., Nomoto, K., Maeda, K. 2005, The First Chemical Enrichment in the Universe and the Formation of Hyper Metal-Poor Stars, Science 309451

[22] Meynet, G., Ekström, S., Maeder, A. 2006, The early star generations: the dominant effect of rotation on the CNO yields, Astronomy and Astrophysics 447623

[23] Piau, L., Beers, T.C., Balsara, D.S., Sivarani, T., Truran, J.W., \& Ferguson, J.W. 2006, , Astrophysical Journal, in press [astro-ph/0603553]

[24] W. Aoki, Neutron-Capture Processes in the Early Galaxy, this volume

[25] W. Aoki, T. C. Beers, N. Christlieb, J. E. Norris, S. G. Ryan, S. Tsangarides, Carbon Enhanced Metal-Poor Stars. I. Chemical Compositions of 26 Stars, Astrophysical Journal, in press 